\title{
Domestic Language Accent Detector Using MFCC and GMM
}

\author{
Roshni Rajan.K \\ Department of Electronics and Communication Engineering, \\ Jyothi Engineering College, Thrissur, Kerala, India.
}

\author{
Annie P Joseph \\ Department of Electronics and Communication Engineering, \\ Jyothi Engineering College, Thrissur, Kerala, India.
}

\begin{abstract}
In day today life, speech is commonly used for communication purpose. So that its recognition has an important role. There are already recognition systems for English and that has been used in different automation and assistance purposes. But in the case of local languages it seems to be difficult in recognition. In the case of Malayalam the usage of words, its pronunciation as well as the meaning vary with respect to the regions. So the same word has different meaning in different areas and hence it become complex. This paper aims to recognize different accents of Malayalam in accounts with the place of the user. Initially a set of training data is acquired from different areas and from the data Mel Frequency Cepstral Co-efficients are extracted as features. A Gaussian Mixture Model is made in accordance with the data which uses Energy Maximization Algorithm.It recognizes the spoken accents and can be used for various home automation and virtual assisting technologies.
\end{abstract}

Keywords: GMM, MFCC

\section{INTRODUCTION}

In this paper speech features have been explored to recognize three major accent of Malayalam used in three different regions Ernakulam, Thrissur and Thiruvananthapuram. The dialect of a given language is a pattern of pronunciation of a language used by the community of native speakers and the speech samples are collected from the native speakers of the different accents for both training and testing. Using Mel Frequency Cepstral Coefficients the features of the each speech samples are extracted for both training and testing. Gaussian Mixture Model (GMM) is used for classification of the speech based on accent and the experiment is implemented in Raspberry Pi.

This paper consists of five sections: Literature review is discussed in Section II. Section III deals with Methodology for the proposed techniques. Section IV illustrates the implementation Section V discusses Results and discussions and conclusion in Section VI.

\section{LITERATURE REVIEW}

Speech recognition has become a practical concept, which is now being implemented in different languages around the world. Speech recognition covers a wide range of real-world human language applications which includes the information recovery also. Speech is considered as the common means of the communication as the information plays the basic role in conversation.

Speech recognition is the conversation of the speech that is captured by a microphone or a telephone is to a set of words. It can have either final output or it can synthesis to pronounce into sounds, which means speech-to-speech. In order to achieve speech understanding, Speech recognition can serves as the input to linguistic processing.

Speech recognition systems are characterized by speaking modes and speaking styles, environmental matters, vocabulary, acoustic model, language model, Signal to Noise Ratio (SNR) etc.The basic model of speech recognition system includes preprocessing, feature extraction and classification as shown in Fig 1.1.

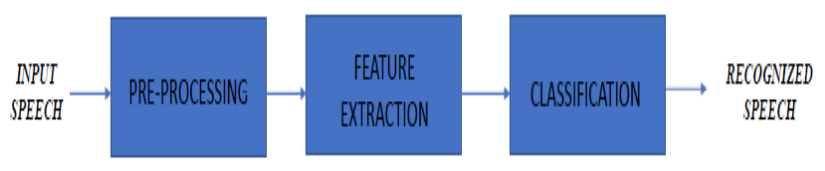

Figure 1.1: Basic model of speech recognition system

At the time of recording speech due to noise signals interference may occurs and it degrades the performance of the system. Before feeding the speech signal to feature extraction block these noise contained in speech signal must be removed. This task is done by pre-processing block. The main function of the feature extraction is to extract the feature from speech signal and to represent them using appropriate data model of the input speech signals. .

\section{METHODOLOGY}

MFCC-GMM based accent recognition system for Malayalam speech signals in MATLAB and Raspberry Pi is used for the implementation. Mel Frequency Cepstral Coefficients (MFCC)[1] are the features used for classification whereas GMM classifier is used for accent recognition.

The speech input to the system is given through a microphone which is connected to the USB port of the Raspberry Pi via a USB sound card. The USB sound card is used to connect 
microphone and speaker to the USB port of any device. Raspberry Pi processes the input speech and compare it with the data used to train the system and predict the best probable accent.The block Diagram for the Proposed System is shown in figure 1.2 .

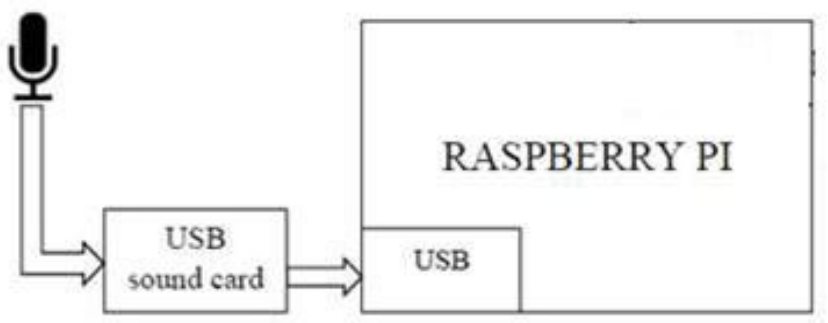

Figure 1.2: Block Diagram of the Proposed System

\section{Speech Processing}

The input speech is processed inside the Raspberry Pi. The accent recognition system is consisting of three processes.

1. Feature extraction

2. The training phase

3. The testing phase

In first process, the voice features acquired from different persons are extracted by using MFCC technique[4]. MFCC is used as the acoustic features of human voice and the human voice pitch is considered in the form of frequencies and scale them on the Mel scale. These features extracted are unique to others. In the training phase, GMM trains the extracted features[5].Expectation and Maximization algorithm is used to train the extracted features of human voice in the system and the data base for the same is created[2]. In the testing phase after the feature extraction the features are compared with the trained database to predict the accent.

Firstly, the speech samples are collected for the training and tested separately. The training samples are taken for the three accents of Malayalam language i.e.Ernakulam (Ekm), Thrissur (Tsr) and Thiruvananthapuram (Tvm). From these training samples MFCC twelve feature average vectors have been extracted for the three accents separately.

GMM training models are built for each of the three accents separately. In the testing phase the testing samples from each of the three regions accents have been taken and the MFCC feature set for each of the test speech signal has been obtained. Then GMM testing is done for the each speech signal.The result of the GMM testing is the recognized accent of the given test speech sample.

\section{MelL Frequency Ceptral Coefficients (MFCCS)}

The first stage of the recognition the input waveform transforms into streams of acoustic feature vectors were each vector represents the information in a small time window of the signal. The concept of the feature extraction is divided into two: first is to compress the speech signals into feature vector; secondly is to choose the useful features which are insensitive to speech variation, changes of environmental conditions etc. However, these speech variation and changes of environmental conditions are crucial in accent recognition system where performance has degraded massively in the case of their existence. Figure 1.3 shows the block diagram of calculating MFCCs.

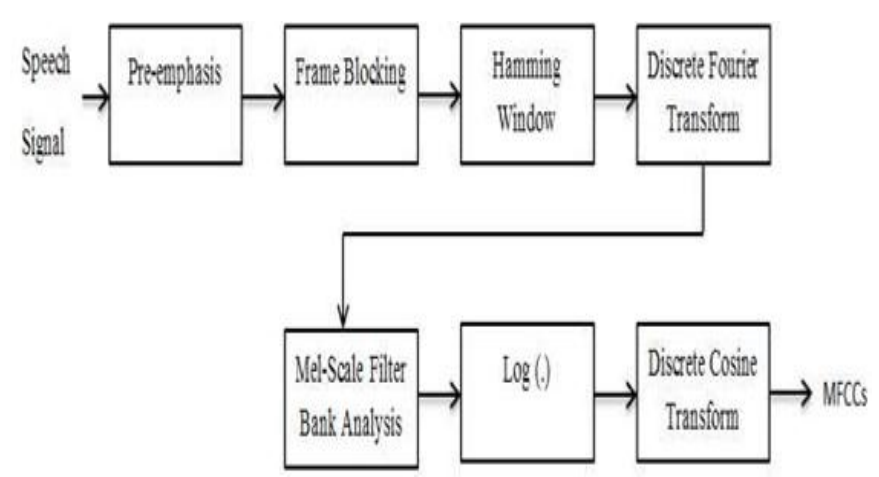

Fig.1.3 shows the block diagram of calculating MFCCs.

\section{IMPLEMENTATION}

The system is implemented in Raspberry Pi and the code is written in python with the help of packages for python and the system is also implemented in MATLAB.

The Raspberry Pi is a series of credit card-sized single-board computers developed in the United Kingdom by the Raspberry Pi Foundation. In this paper Raspberry Pi 3 model B is used. As the Raspberry Pi doesn't have any $3.5 \mathrm{~mm}$ audio input jack the input to the system is given via the USB port of the Pi using a USB sound card. It has USB type-A male connector for connecting with Raspberry $\mathrm{Pi}$, stereo output jack for audio output and mono microphone-input jack for connecting the mic.

MATLAB is a high-level language and interactive environment for numerical computation, visualization, and programming. Using MATLAB, one can analyze data, develop algorithms, and create models and applications. The language, tools, and built in math functions enable you to explore multiple approaches and reach a solution faster than with spreadsheets or traditional programming languages, such as $\mathrm{C} / \mathrm{C}++$ or Java.

\section{RESULTS AND DISCUSSIONS}

A specific sentence is recorded from various people across the three different regions (Thrissur, Ernakulam and Trivandrum). The Figure 1.4. shows a recorded audio sample before processing. For all these audio data, MFCC feature is extracted and a database is made.

For a given input MFCC feature is extracted and compared with all the rest feature data set Gaussian Mixture Model is used as classifier and it uses Expectation Maximisation algorithm. A portion of data set is shown in the Figure 1.5 and the 3D Gaussian Model for a sample is shown in Figure 1.6. 


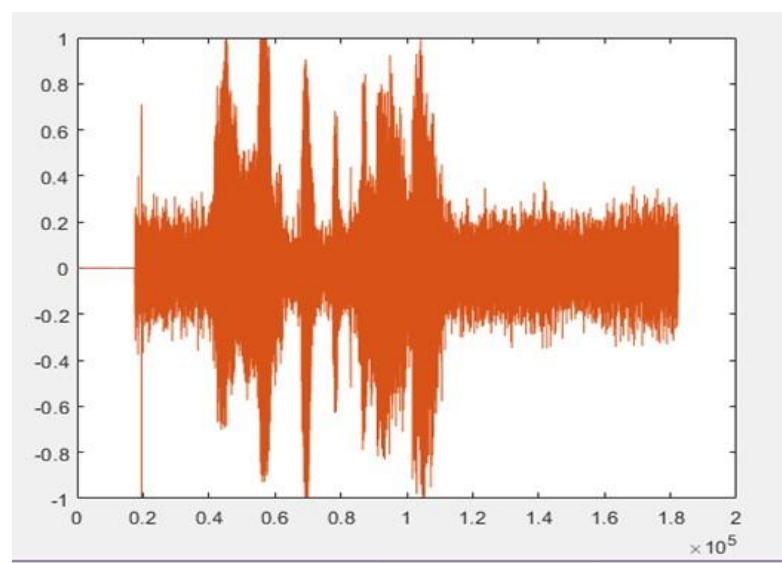

Figure 1.4: Audio Sample

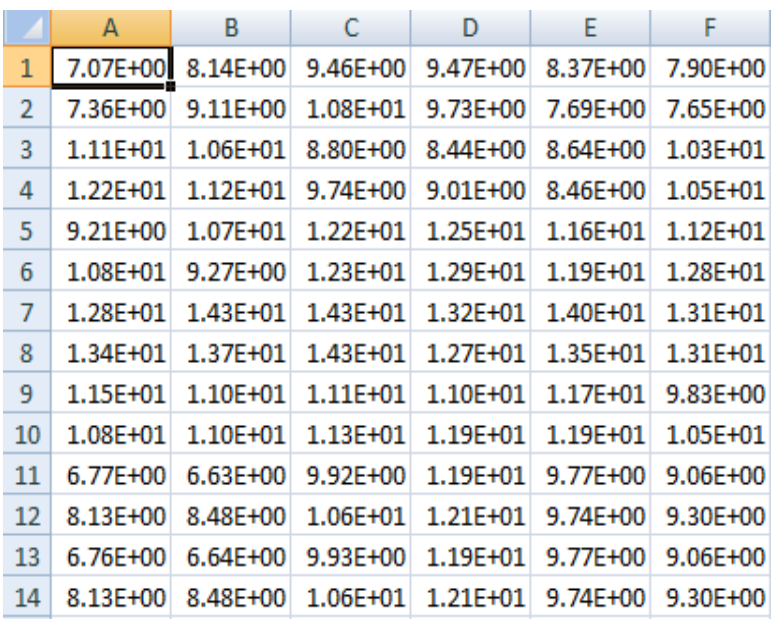

Figure 1.5: MFCC Dataset

The parameters used for feature extraction block implemented in Raspberry Pi are given below

Sampling frequency $=8000 \mathrm{~Hz}$

Frame duration $=25 \mathrm{~ms}$

Frame overlapping $=10 \mathrm{~ms}$

Number of DFT points $=512$

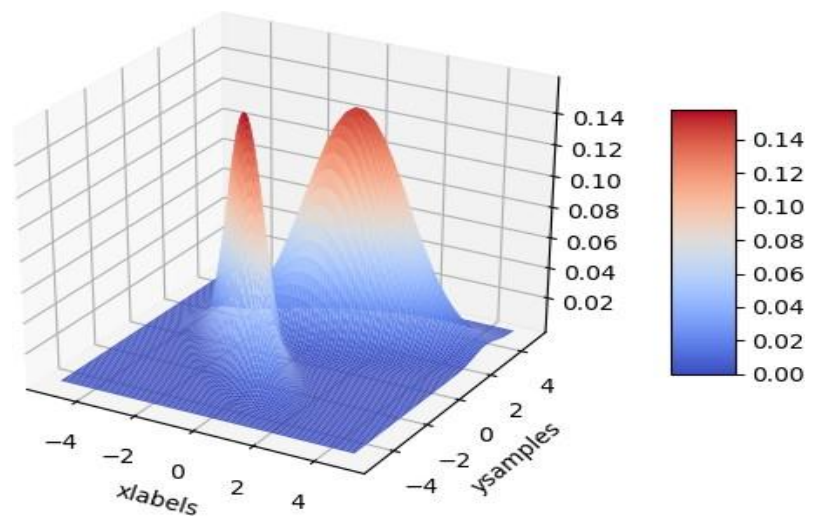

Figure 1.6: 3D Gaussian Mixture Model

\section{CONCLUSION}

This paper implements an accent recognition system using MFCCs and GMM for a Malayalam language in Raspberry Pi. Due to the memory constrains in the handheld device, the system is limited to recognize accent of three districts in Kerala. For the proper functioning of the system, there should be distinct pauses between the words. So the system is a limited vocabulary speaker independent accent recognition system. By using larger training data including utterance from a large number of speakers with variations in age and accent the accuracy can be further improved .

If it is possible to train with large dataset then it can be used in entire state of Kerala.It can be develeped to an application in Mobile phones. It can be used to access virtual systems such as home automation system without knowing English i.e., can access the system with their own local language in Malayalam.

\section{REFERENCES}

[1] Mannepalli, K., Sastry, P. N., Suman, M. (2016). "MFCCGMM based accent recognition system for Telugu speech signals.'International Journal of Speech Technology,19(1), 87-93.

[2] Kurzekar, P. K., Deshmukh, R. R., Waghmare, V. B., Shrishrimal, P.P.(2014). "A comparative study of feature extraction techniques for speech recognition system". International Journal of Innovative Research in Science, Engineering and Technology, 3(12), 18006-18016.

[3] Anusuya, M. A., Katti, S. K. (2011). Classification Techniques used in Speech Recognition Applications: A Review. Int. J. Comp. Tech. Appl, 2(4), 910-954.

[4] Kaur, K., Jain, N. (2015). Feature Extraction and Classification for Automatic Speaker Recognition System-A Review. International Journal of Advanced Research in Computer Science and Software Engineering,

[5] Gupta, S., Pathak, M. A., Saraf, M. A. (2014). A study on speech recognition system: a literature review. International Journal of Science, Engineering and Technology Research, 3(8), 2192-2196.

[6] Gaikwad, S., Gawali, B., Kale, K. V. (2013). Accent Recognition for Indian English using Acoustic Feature Approach. International Journal of Computer Applications, 63(7).

[7] Bhatia, M., Singh, N., Singh, A. (2015). Speaker Accent Recognition by MFCC Using KNearest Neighbour Algorithm: A Different Approach. International Journal of Advanced Research in Computer and Communication Engineering, 4(1).

[8] Sunija, A. P., Rajisha, T. M., \& Riyas, K. S. (2016). Comparative study of differ- ent classifiers for Malayalam dialect recognition system. Procedia Technology, 24, 1080-1088.

[9] Biadsy, F. (2011). Automatic dialect and accent recognition and its application to speech recognition (Doctoral dissertation, Columbia University). 
International Journal of Applied Engineering Research ISSN 0973-4562 Volume 15, Number 8 (2020) pp. 800-803

(C) Research India Publications. https://dx.doi.org/10.37622/IJAER/15.8.2020.800-803

[10] Gholipour, A., Sedaaghi, M. H., \& Shamsi, M. (2012, September). The contribution of prosody to the identification of Persian regional accents. In 2012 IEEE Symposium on Industrial Electronics and Applications (pp. 346-350). IEEE.

[11] Singh, N., Khan, R. A., Shree, R. (2012). Mfcc and prosodic feature extraction techniques: A comparative study. International Journal of Computer Applications, 54(1). 